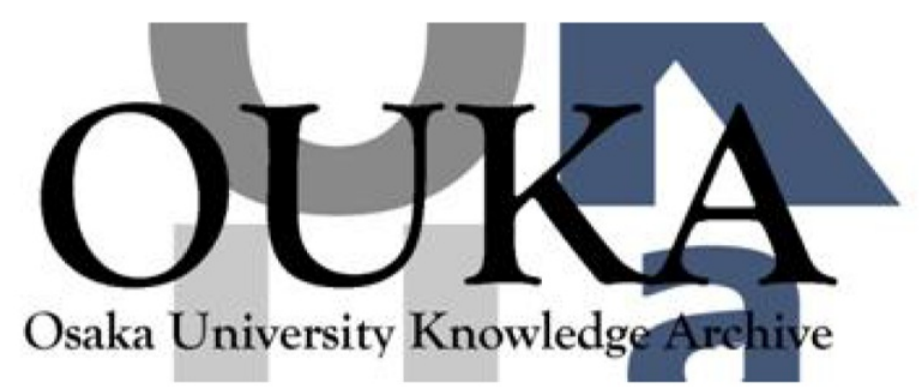

\begin{tabular}{|c|l|}
\hline Title & $\begin{array}{l}\text { Photomodulated electron-spin resonance in } \\
\text { amorphous silicon }\end{array}$ \\
\hline Author(s) & Hattori, K.; 0ta, Y.; Sato, K. et al. \\
\hline Citation & Journal of Applied Physics. 84(9) p. 4974-p. 4978 \\
\hline Issue Date & $1998-11-01$ \\
\hline oaire:version & VoR \\
\hline URL & https://hdl. handle. net/11094/3306 \\
\hline rights & \\
\hline Note & \\
\hline
\end{tabular}

Osaka University Knowledge Archive : OUKA

https://ir. Library. osaka-u. ac. jp/

Osaka University 


\title{
Photomodulated electron-spin resonance in amorphous silicon
}

\author{
K. Hattori, ${ }^{\text {a) }}$ Y. Ota, K. Sato, and H. Okamoto \\ Department of Physical Science, Graduate School of Engineering Science, Osaka University, Toyonaka, \\ Osaka 560-8531, Japan
}

(Received 29 May 1998; accepted for publication 27 July 1998)

\begin{abstract}
Electron-spin resonance (ESR) under a modulated photoexcitation has been measured for investigating the nonequilibrium carriers trapped at band-tail states in hydrogenated amorphous silicon. The photomodulation technique is successfully applied for detecting the weak ${ }^{29} \mathrm{Si}$ hyperfine structures involved in the light-induced ESR spectrum. Access to the recombination kinetics for band-tail carriers is obtained by the frequency-domain study of modulated ESR signal. Physical insights which led from these measurements are discussed quantitatively. (C) 1998 American Institute of Physics. [S0021-8979(98)02121-5]
\end{abstract}

\section{INTRODUCTION}

Hydrogenated amorphous silicon $(a-\mathrm{Si}: \mathrm{H})$ shows two different electron-spin resonance (ESR) lines centered at $g$ $=2.004$ and 2.01 under optical illumination. It is widely accepted that these ESR signals are ascribed to metastable electrons and holes localized in the band-tail states. ${ }^{1}$ The measurements of light-induced ESR (LESR) could offer a quantitative probe for their local environment as well as nonequilibrium statistics. The conventional way to observe the LESR spectra is comprised of the steady-state measurements for extracting light-induced fractions of ESR signal. However, the prevailing simple approach often meets with experimental limitations, mainly due to base-line fluctuations. Specifically, the accurate detection of normally weak absorption tail composed of ${ }^{29} \mathrm{Si}$ hyperfine line is hardly achieved by using this method. The difficulty thus restricts the knowledge derived from the measurements.

The problem of obtaining the measurement over a whole range of LESR spectrum with a sufficient signal-to-noise ratio can be solved by the pulsed-ESR technique, of which excellent capability has been demonstrated recently. ${ }^{2,3}$ An alternative with an experimental ease is thought to be the photomodulation method with use of a lock-in technique, ${ }^{4}$ which is expected to allow a direct measurement of lightinduced signal with the required high sensitivity. Furthermore, the photomodulated ESR (PMESR) technique has a decisive advantage of surveying the dynamic behavior of nonequilibrium carriers that show up in the LESR spectrum, leading to the assessment of relaxation and recombination mechanism. This article presents the results of comprehensive studies performed by using the PMESR technique as well as the physical implications gained for them.

\section{EXPERIMENT}

Two samples of intrinsic rf-glow-discharge $a$-Si:H were prepared for the experiment, one of which was a powdered sample made from a 7- $\mu \mathrm{m}$-thick film deposited on an Al foil substrate and mainly used for obtaining an increased signal-

\footnotetext{
${ }^{\text {a)} E l e c t r o n i c ~ m a i l: ~ h a t t o r i @ e e . e s . o s a k a-u . a c . j p ~}$
}

to-noise ratio. The other sample employed in this study was a 5- $\mu$ m-thick film deposited on a quartz substrate, which permits us to precisely estimate the light-induced spin density as well as the relevant photogeneration rate. The substrate temperature during the film growth was $200^{\circ} \mathrm{C}$. The dark spin density of these samples was measured to be $4-5 \times 10^{15} \mathrm{~cm}^{-3}$.

The PMESR and steady-state LESR measurements were performed at $105 \mathrm{~K}$ in a standard JEOL X-band spectrometer using a microwave power of $100 \mu \mathrm{W}$. No microwave saturation effects were observed under this experimental condition. The ESR signal was monitored in a derivative form by using a magnetic field modulation with an amplitude of 0.3 $\mathrm{mT}$. Samples were illuminated by a $683 \mathrm{~nm}$ light from a diode laser with an intensity in the range between 5 $\mu \mathrm{W} \mathrm{cm}{ }^{-2}$ and $10 \mathrm{~mW} \mathrm{~cm}^{-2}$. In the PMESR measurement, the exciting light was modulated at frequencies varied from 0.2 to $100 \mathrm{~Hz}$. Such a low frequency applied for the measurement ensures that each spin system involved practically stays in thermal equilibrium during the light modulation, owing to a rapid spin-lattice relaxation completed on a time scale of $10 \mu \mathrm{s}$ around $100 \mathrm{~K}^{5}$ A modulated component of the ESR signal was detected by a lock-in amplifier. Both the in-phase and quadrature-phase signals with respect to the photomodulation were obtained with taking a special care for the frequency response of ESR spectrometer we used. The observed PMESR signal depends linearly on the modulated excitation.

\section{RESULTS AND DISCUSSION}

\section{A. Field-Scan Spectra}

Figure 1 displays the field-scan PMESR spectra measured for the powdered sample at light intensity $5 \mathrm{~mW} \mathrm{~cm}^{-2}$ and modulation frequency $1 \mathrm{~Hz}$. It is clearly found that the in-phase $\left(0^{\circ}\right)$ and quadrature-phase $\left(-90^{\circ}\right)$ spectra are both composed of a narrow resonance line on the higher field side and a broad resonance line on the lower field side, as normally observed in the steady-state LESR measurements on $a$-Si:H. According to the conventional interpretation, we shall suppose here that the narrow and broad resonance lines 


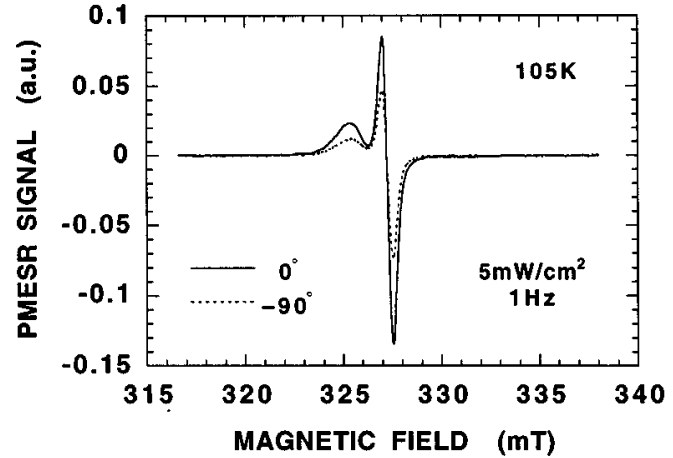

FIG. 1. Field-scan PMESR spectra measured for a powdered sample of undoped $a$-Si:H at $105 \mathrm{~K}$. The background light intensity and the modulation frequency were $5 \mathrm{~mW} \mathrm{~cm}^{-2}$ and $1 \mathrm{~Hz}$, respectively.

arise from nonequilibrium electrons and holes trapped in the band-tail states, respectively. The measured differential spectra have been numerically integrated and converted into the amplitude and phase of absorption. The processed data are presented in Fig. 2. The identical phase for the electron and hole contributions can be readily learned from the figure, pointing out that the amplitude spectrum derived here consists of a simple scalar superposition of their absorption lines. The pulsed-ESR spectrum reported previously ${ }^{2}$ is also plotted for comparison in the figure, where we can clearly see a fairly good agreement in the spectral shape. The spectral similarity was also seen for the PMESR absorption spectrum measured for the sample on quartz.

In the recent work, ${ }^{3}$ Umeda et al. experimentally resolved two overlapping resonance lines by making use of their differing spin-lattice relaxation times, and argued that the high-field-side shoulder mainly originates from the hyperfine interaction between band-tail electron spins and ${ }^{29} \mathrm{Si}$
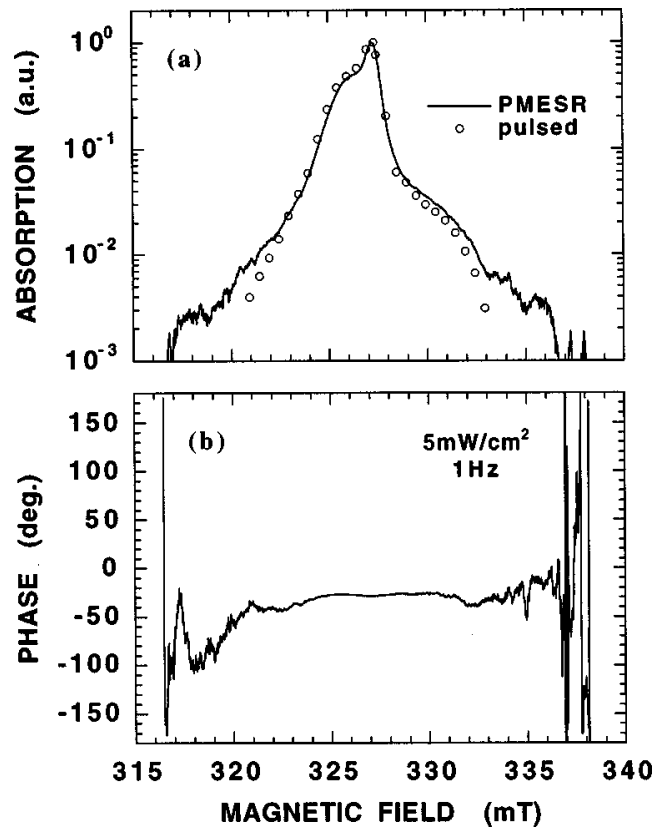

FIG. 2. Amplitude (a) and phase (b) of absorption derived from numerical integration of the derivative spectra presented in Fig. 1. The pulsed-ESR result obtained by Yamasaki et al. (Ref. 2) is shown for comparison. nuclear spins. The area fraction of hyperfine structure $\eta$ can be related, from a statistical consideration, with the number fraction of spin-carrying nuclei $p$ and the number of Si atoms contained in the active region of interaction $m$ by

$$
\eta=1-(1-p)^{m}
$$

for $p \ll 1$. Following this relation, they concluded that a bandtail electron is localized mainly on a couple of $\mathrm{Si}$ atoms ( $m$ $=2.3$ ). Since the present experiments do not offer any precise spectral deconvolution, a simpler but plausible evaluation for the hyperfine structure has been attempted instead.

An equal concentration of the band-tail electrons and holes is likely established for an adequate excitation condition to maintain the charge neutrality. Even in this case, an equal contribution from both types of carriers to the paramagnetic resonance may not be immediately justified, since there is a possibility that a certain number of carriers are spin paired and thus do not show up in the measurement. As described in the literature, an appreciable spin-paired population takes place particularly for the conduction band-tail states in dark equilibrium due to their correlation energy as small as $20 \mathrm{meV} .^{6}$ For the nonequilibrium occupation, the estimation of spin-pairing effect may be essentially altered. Suppose that the band-tail occupation is governed by capturing mobile carriers. The capture probability normally depends on charge state. Therefore, it is logical in this case to consider that an occurrence of doubly occupied states, which necessitates Coulomb-repulsive capture events, is suppressed to a significant degree relative to that of singly occupied states. If a band-tail electron or hole is located very close to each partner with the same charge, a carrier delivery via tunneling could produce the double occupancy to a certain extent. However, the strongly correlated pair is unlikely for a dilute carrier system formed in the present experiment, as implied from the long average inter-center distance in excess of $100 \AA$ estimated from spin density $\sim 10^{17} \mathrm{~cm}^{-3}$. In addition, it should be noticed that the presence of well-isolated carriers is consistent with negligibly weak exchange interaction effects. The absence of significant spin-coupling effects inferred above is indeed trusted from the electron and hole contributions with comparable intensities assessed from the deconvoluted pulsed-ESR spectra. ${ }^{3}$ The spectral analysis for our PMESR results, assuming that one half of the entire absorption is due to band-tail electrons, yields an estimate of the hyperfine structure fraction $\eta=11 \%-16 \%$, indicating that $m=2.4-3.6$ for $p=4.7 \%$.

\section{B. Frequency-resolved spectra}

The frequency-domain measurements enable us to survey the relaxation and recombination processes of nonequilibrium carriers. It is widely accepted that photogenerated carriers quickly thermalize in the band-tail states sufficiently localized in space until they recombine. The thermalization is known to end at a time in the order of $1 \mu \mathrm{s},{ }^{7}$ which corresponds to a frequency scale of around $100 \mathrm{kHz}$ and is far beyond the present experimental range. This means the frequency-resolved PMESR discussed below is recombination controlled. Figure 3 shows the frequency dependence of 


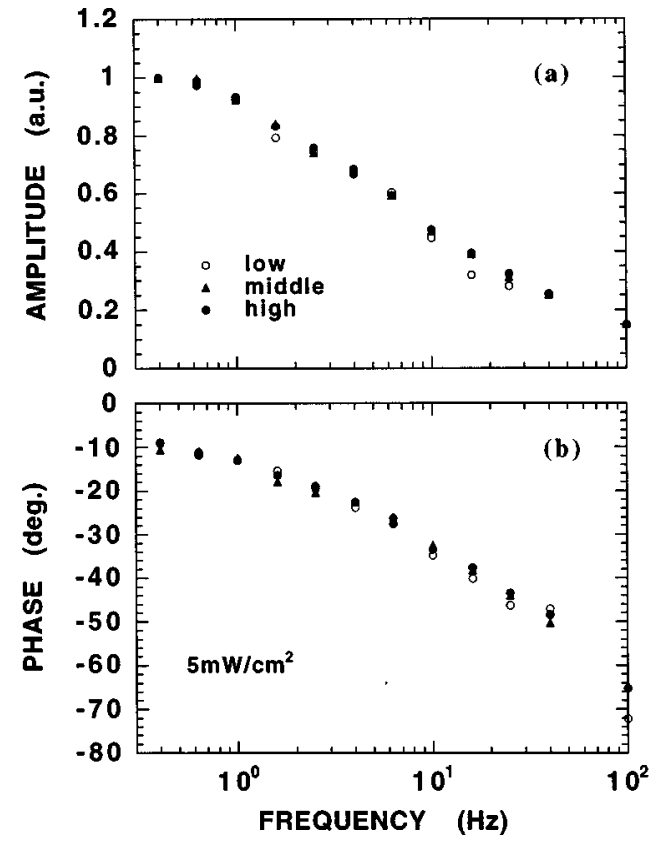

FIG. 3. Frequency dependence of amplitude (a) and phase (b) of PMESR signal measured for a sample of $a-\mathrm{Si}: \mathrm{H}$ deposited on quartz substrate. The measurements were carried out at three fixed magnetic fields where the field-scan derivative spectrum is peaked. The phase value is shifted by $180^{\circ}$ for the higher-field-side negative peak. The measurement temperature and the light intensity were $105 \mathrm{~K}$ and $5 \mathrm{~mW} \mathrm{~cm}^{-2}$, respectively.

amplitude and phase measured for the sample on quartz, at three fixed field positions where the field-scan derivative spectrum is peaked. It is evident in the figure that the amplitude and phase spectra are quite similar irrespective of the probe positions. This feature has also been found for the powdered sample and for various light intensities. The observation is accounted for only by assuming that a direct tunneling recombination prevails for a pair of band-tail electron and hole.

Related measurements have also been made to test this interpretation. A possible contribution from dangling bond defects that are known to be dominant nonradiative recombination centers in $a-\mathrm{Si}: \mathrm{H}^{8}$ has been explored by the lightinduced degradation of sample. No discernible change in the LESR spin density as well as in its light intensity dependence was observed even when the dark spin density was increased by light soaking up to $4 \times 10^{16} \mathrm{~cm}^{-3}$, which is an order of magnitude larger than the initial density. The LESR observation is compatible with the fact that the lowtemperature photoluminescence is independent of the defect density in the range smaller than $10^{17} \mathrm{~cm}^{-3}$. ${ }^{8}$ We therefore conclude that defect-related processes are ruled out from the most probable recombination events. The photoconductivity measurement has also been performed to examine the recombination processes associated with mobile carriers. The light intensity dependence of photoconductivity exhibits a power law behavior with an exponent of about 0.5 , which is a common feature seen in a temperature region around $100 \mathrm{~K} .{ }^{9}$ On the other hand, the LESR spin density depends much more weakly on the intensity. The power law exponent found for our samples is as low as 0.17 , which agrees with the previous measurements showing $0.15-0.2 .{ }^{10}$ Obviously, the LESR re-

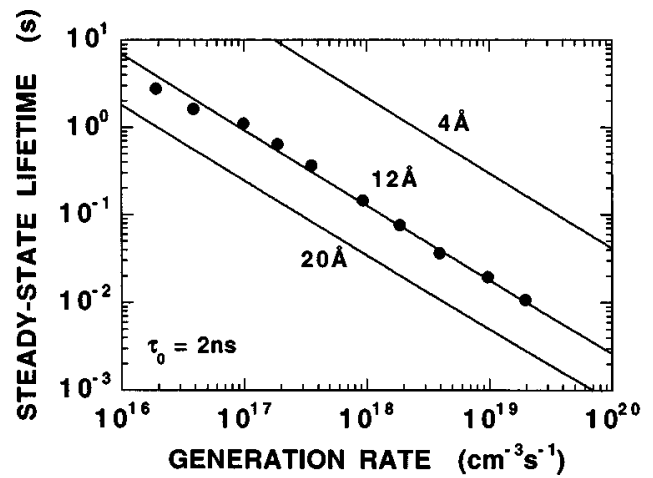

FIG. 4. Steady-state lifetime data obtained from LESR measurements as a function of photogeneration rate. Solid lines represent theoretical plots for various localization lengths $R_{0}=4,12$, and $20 \AA$. The prefactor $\tau_{0}=2 \mathrm{~ns}$ was assumed for the calculation.

sult is not in favor of the bimolecular recombination with mobile carriers. Assuming tail-to-tail tunneling recombination is therefore firmly validated.

A quantitative argument on the recombination kinetics of the nonequilibrium carriers is brought about by determining their lifetime. The steady-state LESR measurement provides an average lifetime $\langle\tau\rangle$ which is simply given as carrier density $N$ divided by photogeneration rate $G$. The lifetime data derived from our LESR measurements are plotted against the photogeneration rate in Fig. 4. The weak variation of spin density mentioned above leads to the lifetime decreased strongly with the generation rate as $G^{-0.83}$, as illustrated in the figure. Access to lifetime is also gained by analyzing a quadrature part of modulated signal, in accord with the procedure of the so-called frequency-resolved spectroscopy technique, which is usually adapted for the investigation of photoluminescence mechanism. A detailed explanation of the basis of the method is given by Depinna and Dunstan $^{11}$ and need not be repeated here. Figure 5 illustrates the quadrature-PMESR spectra measured for two different light intensities, 5 and $0.5 \mathrm{~mW} \mathrm{~cm}{ }^{-2}$. They represent a distribution of lifetime and exhibit a bump which shifts to shorter times as the excitation intensity increases. As indi-

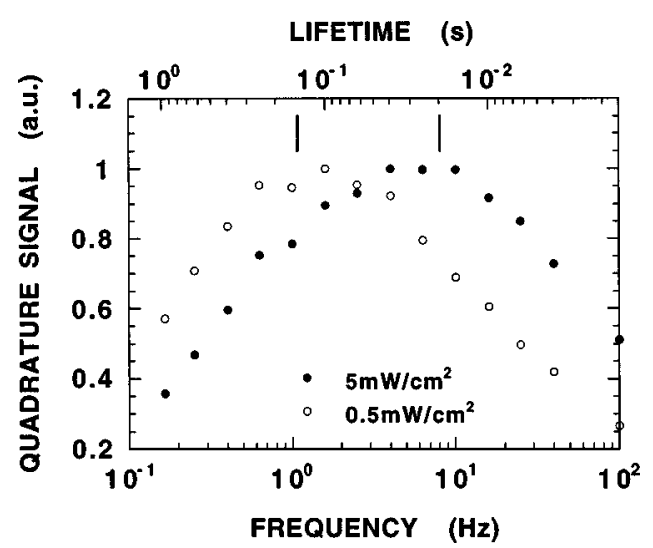

FIG. 5. Frequency-resolved quadrature spectra measured for a sample of $a$-Si:H deposited on quartz substrate with two different light intensities 5 and $0.5 \mathrm{~mW} \mathrm{~cm}^{-2}$. The upper horizontal axis is scaled with lifetime using the relation $\omega \tau=1$. The vertical bars in the figure indicate the steady-state lifetime values. 
cated in the figure, the peak lifetime $\tau_{p}$ coincides with the average lifetime $\langle\tau\rangle$. Physical implications from these measurements will be discussed below in detail.

\section{Recombination model}

We here base the discussion on the assumption that the dominant recombination channel is tail-to-tail tunneling between localized electron-hole pairs. The lifetime of these pairs is related to the intra-pair separation $R$ by $^{12}$

$$
\tau(R)=\tau_{0} \exp \left(\frac{2 R}{R_{0}}\right),
$$

where $R_{0}$ denotes the effective Bohr radius or localization length, and $\tau_{0}$ is a prefactor. The appropriate value of $R_{0}$ is the larger of the values for both types of carriers. The hole is believed to more localized than the electron in $a-\mathrm{Si}: \mathrm{H}$ so that $R_{0}$ can be regarded as the localization length of the electron. Although the above expression, assuming that wave function decays isotropically as $\exp \left(-R / R_{0}\right)$, might be oversimplified, it has been widely applied and is probably valid for an amorphous semiconductor, especially in the case of a sufficiently large $R$. The principle distinction between radiative and nonradiative tunneling transitions lies in the value of $\tau_{0}$. For the radiative transitions, $\tau_{0}$ is assumed to be $10^{-8} \mathrm{~s}$, corresponding to typical dipole transitions. For the nonradiative transitions accompanied by phonon emission, $\tau_{0}$ is thought to be on the order of $10^{-12} \mathrm{~s}$. In general, both types of transitions can occur. In a temperature range around 100 $\mathrm{K}$, the effective prefactor that takes into account the parallel effects of radiative and nonradiative processes is estimated to be $\tau_{0}=2 \times 10^{-9} \mathrm{~s}$ from the photoluminescence quantum efficiency data. ${ }^{9,13,14}$

From the tunneling rate $\tau^{-1}$ decreasing exponentially with $R$, it is assumed that recombination preferentially takes place between the nearest available neighbors. Every photogenerated electron is surrounded by its geminate hole with which it may recombine. The geminate kinetics ${ }^{12}$ is independent of the carrier density, and plays a crucial role in recombination as the background carrier concentration is sufficiently low such that there is no inter-pair interaction. The relevant lifetime distribution reflects the random walk of band-tail carriers during thermalization. For a moderately high concentration, on the other hand, the probability for an electron to recombine with uncorrelated hole increases and eventually a distant-pair tunneling dominates recombination kinetics. The distant-pair kinetics ${ }^{15}$ expects that an increase in photogeneration rate leads to a decrease in the pair separation and hence, according to Eq. (1), the recombination lifetime becomes shorter. The LESR and PMESR behaviors observed here indicate that the band-tail carriers undergo the distant-pair recombination.

The steady-state balance equation can be written in the form

$$
N=G\langle\tau\rangle .
$$

Here, the average lifetime $\langle\tau\rangle$ is given with the distribution function of the pair separation $P(R)$ by

$$
\langle\tau\rangle=\int_{0}^{\infty} P(R) \tau(R) d R .
$$

It is also expected that the frequency-dependent quadrature signal $S_{-90^{\circ}}$ is expressed as a superposition of single response function $\omega \tau /\left(1+\omega^{2} \tau^{2}\right)$

$$
S_{-90^{\circ}} \propto \int_{0}^{\infty} P(R) \tau(R) \frac{\omega \tau(R)}{1+\omega^{2} \tau^{2}(R)} d R,
$$

where $\omega$ denotes the angular frequency. The average lifetime and the peak lifetime determined from Eqs. (3) and (4) are both intimately correlated with the distribution functions $P(R)$ for recombining pairs. An important indication from the present observation that both lifetime values coincide with each other is that the corresponding distribution is sharply peaked. Using a delta function approximation $P(R)=\delta(R$ $-\bar{R}$ ), one obtains a simplified expression

$$
\langle\tau\rangle=\tau_{p}=\tau_{0} \exp \left(\frac{2 \bar{R}}{R_{0}}\right) .
$$

As the most probable separation, here we take $\bar{R}$ $=(1 / 2 \pi N)^{1 / 3} .{ }^{9}$ The light intensity dependence of lifetime can be calculated according to Eqs. (2) and (5). The results of calculation for various localization lengths $R_{0}$ are plotted by solid lines in Fig. 4. An experimentally observed strong variation with the generation rate can be reasonably accounted for by the present model, as illustrated in the figure. The theoretical result for $R_{0}=12 \AA$, which is consistent with the photoluminescence estimates $11-12 \AA{ }^{7,8}$ gives the best fit to our data.

The narrow distribution assumed here is contrastive to the theoretical prediction for a distant-pair luminescence by Dunstan, ${ }^{15}$ showing a substantial distribution of pair separation. However, it must be stressed here that assuming any broad distributions is confronted with serious problems. In fact, it is analytically confirmed that the pair distribution for a randomly distributed collection of carriers ${ }^{12}$ leads to orders of magnitude difference between two lifetime values, and that the Gaussian distribution is applicable for the interpretation only when its standard deviation is smaller than the localization length. The narrow distribution thus forms a good approximation for $\langle\tau\rangle$ and $\tau_{p}$, while its extreme limit $P(R)=\delta(R-\bar{R})$ is not suited for illustrating the measured spectral shape of quadrature PMESR. Further studies are clearly needed for deriving the proper representation of distribution function as well as for obtaining the rigorous description of the distant-pair system.

In Sec. III A, the analysis of hyperfine structure concludes that a band-tail electron is mainly localized on a few $\mathrm{Si}$ atoms, suggesting that its probability density falls off rapidly on an atomic scale. This comes into conflict with the localization length $R_{0}=12 \AA$ estimated for weakly coupled recombining pairs with $R \geqslant 100 \AA$, indicating that a single exponential decay of wave functions does not retain its validity for a short-range behavior. ${ }^{16}$ The full understanding of band-tail electronic states is not achieved at the present stage of investigation and is a subject of further studies. 


\section{SUMMARY}

We have applied the PMESR spectroscopy technique for the investigation of nonequilibrium band-tail carriers in $a$ $\mathrm{Si}: \mathrm{H}$. Use of this technique allows us to acquire the accurate measurements of wide-dynamic-range LESR spectra. The PMESR measurement of ${ }^{29} \mathrm{Si}$ hyperfine lines agrees well with the previous pulsed-ESR result, leading to an assessment that a band-tail electron is mainly localized on a few $\mathrm{Si}$ atoms. Frequency-domain study of PMESR yields a quantitative argument on recombination kinetics for the band-tail carriers. The experimental observation is indicative of a distant-pair tunneling recombination between the localized electron and hole. The long-range electron wave function with decay length of $12 \AA$ is suggested from the lifetime measurements.

${ }^{1}$ R. A. Street and D. K. Biegelsen, Solid State Commun. 33, 1159 (1980). ${ }^{2}$ S. Yamasaki, H. Okushi, A. Matsuda, and K. Tanaka, Phys. Rev. Lett. 65, 756 (1990).

${ }^{3}$ T. Umeda, S. Yamasaki, J. Isoya, A. Matsuda, and K. Tanaka, Phys. Rev. Lett. 77, 4600 (1996).

${ }^{4}$ J. Hautala and J. D. Cohen, J. Non-Cryst. Solids 164-166, 371 (1993).
${ }^{5}$ M. Stutzmann and D. K. Biegelsen, Phys. Rev. B 28, 6256 (1983).

${ }^{6}$ G. Schumm, W. B. Jackson, and R. A. Street, Phys. Rev. B 48, 14198 (1993).

${ }^{7}$ C. Tsang and R. A. Street, Phys. Rev. B 19, 3027 (1979).

${ }^{8}$ R. A. Street, J. C. Knights, and D. K. Biegelsen, Phys. Rev. B 18, 1880 (1978).

${ }^{9}$ J.-H. Zhou and S. R. Elliott, Phys. Rev. B 48, 1505 (1993).

${ }^{10}$ M. Bort, W. Fuhs, S. Liedtke, R. Stachowitz, and R. Carius, Philos. Mag. Lett. 62, 227 (1991).

${ }^{11}$ S. P. Depinna and D. J. Dunstan, Philos. Mag. B 50, 579 (1984).

${ }^{12}$ R. A. Street, in Semiconductors and Semimetals, edited by J. I. Pankove (Academic, New York, 1984), Vol. 21, Pt. B, pp. 197-244.

${ }^{13}$ T. S. Nashashibi, I. G. Austin, and T. M. Searle, Philos. Mag. 35, 831 (1977).

${ }^{14}$ W. B. Jackson and R. J. Nemanich, J. Non-Cryst. Solids 59,60, 353 (1983).

${ }^{15}$ D. J. Dunstan, Philos. Mag. B 46, 579 (1982).

${ }^{16}$ Assuming a single exponential envelope of wave function, the localization volume defined by the radius $R_{0} / 2=6 \AA$ contains about two ${ }^{29} \mathrm{Si}$ atoms for $p=4.7 \%$. Most likely they are incorporated in a normal $s p^{3} \mathrm{Si}-\mathrm{Si}$ bonding network. The contact interaction then leads to strong hyperfine lines. For instance, an equivalent interaction for two nuclear spins expects three separated lines with 1:2:1 intensity ratio. The relative weight of hyperfine satellites is $\eta=50 \%$. A significant spectral broadening would also be caused by a random distribution of ${ }^{29} \mathrm{Si}$ nuclei. The suggested features clearly contradict the observation. 